$$
\text { [Stellarator theory] }
$$

\title{
Progress Report
}

This section is concerned with work which has already been done, modeling divertors and edge-plasmas at a kinetic level.

In the last few years, we have developed a novel approach to solving kinetic equations. The method we use has become more sophisticated, and a vatiety of techniques have been devised to accelerate the calculation. We began by using particle simulations, but switched to solving the kinetic equation (albeit using particle 'concepts') when we found statistical 'noise' in particle simulations to be a severe problem. We thus work directly with a distribution function, which is a smooth function and does not require averaging of any kind to make it more tractable. We refer to our method as a "convective scheme" (cs) see, e.g. ref.II.1.; it will be described, briefly, in what follows.

Figure II.1 provides the basis for describing the algorithm. The idea of the convective scheme (CS) as applied to this problem is, given a suitable numerical mesh throughout phase space, to move the particles associated with the individual cells of the mesh to a new location based on their initial coordinates (position and velocity), the fields present (here assumed to be entirely electrostatic), the collision probability, and the time step. The particles are then distributed into cells of the mesh based on the phase space overlap as indicated in the upper portion of Fig.II.1. In the limit of infinitely small mesn cells, the initial particle density in a single cell resembles a delta function in phase space, and hence there is a connection between the cs and Green's functions or propagators. The solution of kinetic equations by this method is straightforward because the appropriate propagator is intuitively obvious, at least for time steps much less than a mean collision time.

To speed up the kinetic calculation, a fluid-like calculation has been developed which uses transport coefficients derived from the kinetic calculation.

We calculate the flux out of each cell, through both faces of the cell, $\Gamma_{\text {out }}$ (left) and $\Gamma_{\text {out }}$ (right). The flux into the cell from 
the neighboring cell is not included in this. In essence, we equate the directed part of the flux to a mobility-term.

We set

$$
\Gamma_{\text {out }}(\text { right })-\Gamma_{\text {out }}(\text { left })=n \mu E
$$

whereas

$$
1 / 2\left(\Gamma_{\text {out }}(\text { right })+\Gamma_{\text {out }}(\text { left })\right)=D
$$

If only one cell has a non-zero population, i.e. if we consider a cell in isolation, as we did in defining the fluxes,

$$
\frac{\partial n}{\partial x}= \pm \frac{n}{\Delta x}
$$

$\mu$ and $D$ for the cell are defined by these equations. They are found from the kinetic code as functions of space (and time, if necessary) and can subsequently be used in a fluid simulation.

The fluid calculation and the kinetic calculation are run alternately, the former providing speed and the latter accuracy, until a converged solution is obtained.

The other model described in this proposal is also a kinetic, numerical edge plasma model. It is a particle simulation, developed by Hussein and Emmert, and being applied to various stellarator divertor calculations by Dr. Hussein. Ions are "followed" by the code; the electrostatic potential is found from the ion density, using an assumption of quasineutrality and assuming a Boltzmann distribution of electron density. This model is presented in detail in section III.

The physics objectives of our program of divertor modeling are overall quite broad, since the fluid models which are frequently used in divertor studies are highly suspect and so the whole issue of divertor performance needs to be carefully reassessed. In the short term, however, we have specific goals related mainly to stellarator divertor performance and the interpretation of available data, especially from IMS.

Unlike the particle-following work described in the next section, the cs code was developed to provide accurate calculations 
of ion and electron distribution functions. Even when augmented by a fluid code, the enormity of the computational problem being solved means that the CS is slower than the particle simulation.

The type of problem that should be addressed with it is also somewhat different. Large numbers of runs to test performance of a divertor in detail are best done by the particle code. The cs/fluid code is being employed to examine more fundamental issues, as indicated below.

The CS is also being developed in order to interface with a fluid code such as that of Braams [II.2]. In this context, the full CS is needed to define the validity of the fluid model used. For some applications, a limited model with kinetic ions and Boltzmann electrons and a self-consistent electrostatic potential (exactly as in the particle simulation) will be very fast and effective.

As an example, we can consider the issue of ambipolar potential formation. It is often assumed that as the ion collision frequency (and, in particular, the charge-exchange collision frequency) goes up, then the ambipolar potential must grow stronger to accelerate ions to the Bohm velocity. Recently, Hussein has studied a presheath contained in a bundle of magnetic field lines which expand as the wall is approached. He found the opposite result; the effect of collisions was to flatten the density profile, in the presence of the magnetic field. (We shall give a simple analytic treatment which explains this result, shortly. His numerical method is described in the next section.) This is of considerable importance, in that a flat density profile in a collisional plasma means low ambipolar potentials and low velocities. The impact of this on divertor performance could be very significant. To see what are the main issues which need to be studied further, we now give a brief derivation which captures the main physical effects present in Hussein's calculations. First results from Hussein's code are given in a paper in the Appendix of the proposal. 
We assume:

a) The bundle of field lines has an area $A \alpha 1 / B$, where $B$ is the magnetic field strength; $B=B_{d} /(1+\alpha x)$ implies a linear increase in $A$ with distance $x$.

b) The total particle flux $\Gamma A=$ Constant, so the flux per unit area is

$$
\Gamma=n v=\frac{\Gamma_{o}}{1+\alpha x}
$$

where $\Gamma_{0}$ is the maximum value of the flux per unit area $\Gamma$, at $x=0$.

c) The electron density has a Boltzmann distribution,

$$
n_{e}=n_{0} \exp (e \Phi / k T)
$$

and the presheath is quasineutral, $n_{i}=n_{e}$.

d) Ion motion is dominated by charge exchange collisions, which cause the particles to essentially stop. The mean velocity is thus $v=1 / 2$ at, where $a$ is the acceleration and $t$ is the mean time between collisions,

$$
t=\frac{\pi \lambda}{2 a}
$$

$\lambda$ being the mean free path. This implies

$$
v^{2}=\frac{\pi \lambda a}{8}=\frac{\Gamma_{0}}{n(1+\alpha x)}
$$

From (1),

$$
\frac{\partial n}{\partial x}=\frac{n e}{k T} \frac{\partial \phi}{\partial x}=-\frac{n m}{k T} a
$$

\section{DISCLAIMER}

This report was prepared as an account of work sponsored by an agency of the United States Government. Neither the United States Government nor any agercy thereof, nor any of their employees, makes any warranty, express or implied, or assumes any legal liability or responsibility for the accuracy, completeness, or usefulness of any information, apparatus, product, or process disclosed, or represents that its use would not infringe privately owned rights. Reference herein to any specific commercial product, process, or service by trade name, trademark, manufacturer, or otherwise does not necessarily constitute or imply its endorsement, recommendation, or favoring by the United States Government or any agency thereof. The views and opinions of authors expressed herein do not necessarily state or reflect those of the United States Government or any agency thereof. 
Using (2) to elimirate the acceleration,

$$
-\frac{1}{n} \frac{\partial n}{\partial x} \cdot \frac{\kappa T}{m}=\frac{8}{\pi \lambda} \frac{\Gamma_{0}}{n(1+\alpha x)}
$$

with

$$
\begin{gathered}
\gamma=\frac{16 m \Gamma_{0}^{2}}{\kappa T \pi \lambda}, \\
\frac{\partial\left(n^{2}\right)}{\partial x}=-\frac{\gamma}{(1+\alpha x)^{2}}
\end{gathered}
$$

so

$$
\begin{aligned}
& n^{2}=A-\frac{\gamma / \alpha}{1+\alpha x} \\
& =n^{2}(0)-\frac{\gamma x}{1+\alpha x}
\end{aligned}
$$

This result illustrates the effect of the simple model field, and its expansion. As $x$ becomes large, the second term goes to $\gamma / \alpha$. If $\alpha=0$, however, the density continues to drop instead of going to an asymptote.

The key assumptions in this treatment, which need to be tested, are quasineutrality (and what happens in the sheath) and the Boltzmann distribution of electrons. The fully kinetic treatment is needed to study these effects. This is described more fully in section IV of the proposal. 


\section{Particle Kodeling of stellarator Divertors}

We now turn to the other numerical approach we have developed and to the physics issues to be addressed by means of it.

We summarize the method here; more detail is given in the main proposal. Some early results are in the paper in the Appendix of the proposal.

\section{Particle studies of stellarator Divertors}

It is well known that the stellarator family of toroidal confinement systems possesses a natural divertor [III.1]. This divertor is characterized by a magnetic separatrix, which isolates the confinement region of closed nested toroidal flux surfaces from the surrounding divertor region and the walls of the device. Plasma diffusing out of the main confinement region enters the scrape-off zone and flows along the diverted field lines into the divertor chamber, where it strikes the target plate. Figure III.1 shows the variation of the magnetic field strength along one field line in ATF. The region between the last peak in $|B|$ and the target plate represents the fishtail of the stellarator divertor. A field line typically makes several toroidal transits in the scrape-off layer outside the last closed magnetic surface before entering the fishtail region. The magnetic mirror ratio is typically 2-3 in ATF and the connection length between mirror peaks is short. When in the fishtail region, the field line travels very quickly to the target plate in a region of rapidly dropping magnetic field strength (see Fig.III.1). For reasonable values of the plasma density and temperature in the edge region of current stellarators, the ions are collisional in the scrap-off layer, but are collisionless in the much shorter fishtail region, where the expanding magnetic field can play a significant role on the ion dynamics.

From magnetic ficld line calculations, a large variation of |B| along a field line has been computed in ATF [III.2]. This could lead to significant potential variation along the oper field line. Evaluation of this potential is important for divertor 
calculations. The exact profile of the variation of $|B|$ is needed if a collisional plasma is being considered. As shown in Fig.III.2, in the case of a collisionless plasma it is the overall magnetic field mirror ratio that matters, which means that different magnetic field profiles with the same overall mirror ratio will produce the same potential drop. This is simply because all ions emitted at $x=0$ experience the effect of the same mirror ratio, namely $B(x=0) / B(x=L)$. In the presence of ion-neutral collisions this is no longer true.

Particles streaming out of the main plasma along the diverted field lines into the fishtail region are modeled by a single particle flux source at $x=0$. As illustrated in Fig. III.3, the region $0<x<L$ is sourceless if neutral gas recycling at the neutralizer plate is neglected. Plasma is assumed to be maintained by a hot particle source at $x=0$. In a real experiment, the input particle flux to the edge region is not a constant but rather determined by the confinement time, and fueling requirements.

\section{Formulation and Computational Method}

Plasma flow along the divergent magnetic field of the fishtail region is determined by quasi-neutrality (outside the sheath region),

$$
n_{e}(x)=q n_{i}(x),
$$

where $n_{e}$ and $e$ are the density and charge of the electrons, and $n_{i}$ and $q$ are the density and charge of the ions. We assume the electrons are sufficiently collisional that they can be represented by a Boltzmann distribution

$$
n_{\theta}(x)=n_{\theta 0} \exp \left(\frac{e \Phi(x)}{k T_{\theta}}\right)
$$

where $\Phi(x)$ is the electrostatic potential, $n_{e o}$ is the electron density at $x=0$ where $\Phi$ is defined to be zero, and $k$ is the Boltzmann constant. 
The ion density is computed using a Monte Carlo procedure where a set of test ions is followed until they hit the divertor plate. The time they spend in a given volume element determines the ion density, as described below. This can be thought of as an indirect solution of the Boltzmann equation.

\section{Treatment of Ion-Neutral Collisions}

In the fishtail region the electron temperature, $T_{e}$, is only a few ev which is well below the threshold for electron impact ionization of the working gas, which is assumed to be hydrogen in our simulation. Thus, only charge exchange and elastic scattering collisions between ions and neutrals are considered. The collision dynamics is studied in a three dimensional velocity space using a Monte Carlo treatment [III.3] and assuming an isotropic scattering in the center of mass system. The velocity components of a neutral particle at a collision site are chosen randomly. The parallel velocity component of the interacting ion is determined from its motion along a field line while the perpendicular component is 2 computed from the invariance of the magnetic moment $\mu$.

\section{Numerical Algorithm}

Instead of calculating the ion density of the Boltzmann equation, our approach is to determine the ion density indirectly by computing ion trajectories in the self-produced electostatic potential and the externally applied magnetic field. Plasma streaming out of the SOL is modeled by a single ion source at $x=0$ which emits ions with random velocities sampled from a cumulative probability function, $c(u)$, given by

$$
C(v)=\int_{0}^{v} v^{\prime} f_{i}\left(v^{\prime}\right) d v^{\prime}
$$

where $f_{i}$ is the collisionless ion distribution function in a constant magnetic field as computed at the edge of the source sheath by Emmert et al. [III.4] This is equivalent to giving the 
source a spatial dimension along which ions are born at different points creating a presheath potential drop inside the source itself. Ions born at different points in the source region are accelerated by this source presheath potential to different energies producing an ion energy spread at the end of the source region. We divide the computational region, as shown in Fig. III.3, into cells of width $\Delta \mathbf{x}_{j}$, and assume the acceleration is constant in each cell. The collisionless motion of an ion in the electrostatic potential $\Phi(x)$ and the externally applied magnetic field is described by

$$
m_{i} \frac{d v_{1}}{d t}=\left(-q \frac{d \Phi}{d x}-\mu \frac{d B(x)}{d x}\right)
$$

and

$$
\frac{d x}{d t}=v_{1}
$$

where $x$ is the coordinate along a field line, $B(x)$ is the vacuum magnetic field strength, $v_{1}$ is the ion velocity along the field line, and $\mu$ is the magnetic moment of an ion. starting with an initial guess for the potential profile and the given spatial profile of the applied magnetic field, ions are emitted from the source and their trajectories are computed using the above equations in the region $0<x<L$. The time an ion spends in every cell is then calculated.

If ion-neutral collisions are to be included, ion trajectories are computed from the exact equations of motion rather than the above guiding center equations. Such a detailed ion trajectory is needed because the probability of an ion-neutral collision depends directly on the length of the ion path. The path length estimator method [III.5] is used to determine if a collision event will occur in any given cell. 
The ion density in the $j^{\text {th }}$ cell is then given by

$$
n_{j}=\frac{1}{N} \sum S \frac{\Delta t_{j_{k}}}{\Delta x_{j}} \frac{B\left(x_{j}\right)}{B_{0}},
$$

where $t_{j k}$ is the time spent in the $j^{\text {th }}$ cell of width $\Delta x_{j}$ by the $k^{\text {th }}$ particle. The term $B\left(x_{j}\right) / B_{0}$ models the expansion of the magnetic flux tube as the particles follow the magnetic field. $N$ is the total number of particles emitted from the source. A trajectory is terminated and a particle is lost whenever it hits the divertor plate at $x=L$, which is assumed to be electrostatically floating and perfectly absorbing. The ion density is used in the quasineutrality relation, and the electrostatic potential $\Phi_{j}$ is then determined from the Boltzmann relation. We iterate until a converge potential profile is reached.

It should be noted that the boundary at $x=L$ is placed on the plasma side of the divertor plate sheath edge. The simulation does not enter the sheath itself, which is assumed to be ion attracting. since the divertor plate is electrostatically floating, the requirement of equal ion and electron fluxes to the wall determines $\Phi_{f}$. That is,

$$
\Phi_{f}=\frac{k \mathbf{T}_{\theta}}{e} \ln \left[\frac{\Gamma_{i}}{n_{\theta 0}} \quad \frac{2 \pi m_{\theta}}{k T_{\theta}}\right]
$$

where $m_{e}$ is the slectron mass and $\Gamma_{i}$ is the ion flux at the sheath edge.

\section{References}

[II.1] D.J. Koch and W.N.G. Hitchon, Phys. Fluids B1, 2239 (1989).

[II.2] B.J. Braams, 'The Multifluid Code for simulation of the Edge Plasma in Tokamaks', NET Report NR 68, January 1987. 
[III.1] J. Derr and L. Shohet, IEEE trans. on Plasma Phys. PS-9, 234 (1981).

[III.2] J. Rome, R. Fowler and G. Emmert, Divertor Calculations for stellarators, Int. Stellarator/Heliotron workshop, Kyoto, Japan (1986).

[III.3] E.D. Cashwell and C.J. Everett, Monte Carlo Method for Random Walk Problems, pp. 56-62 (Pergamon, 1959).

[III.4 G. Emmert, R. Wieland, A. Mense and J. Davidson, Phys. Fluids 34,803 (1980).

[III.5] M. Hughes and D. Post, J. Comput. Phys. 28, 43 (1978).

[III.6] G. Emmert, UWFDM-343 (1980), Fusion Technology Institute, University of Wisconsin-Madison.

[III.7] A. Bailey and G. Emmert, Nucl. Fusion 24, 1439 (1984). 


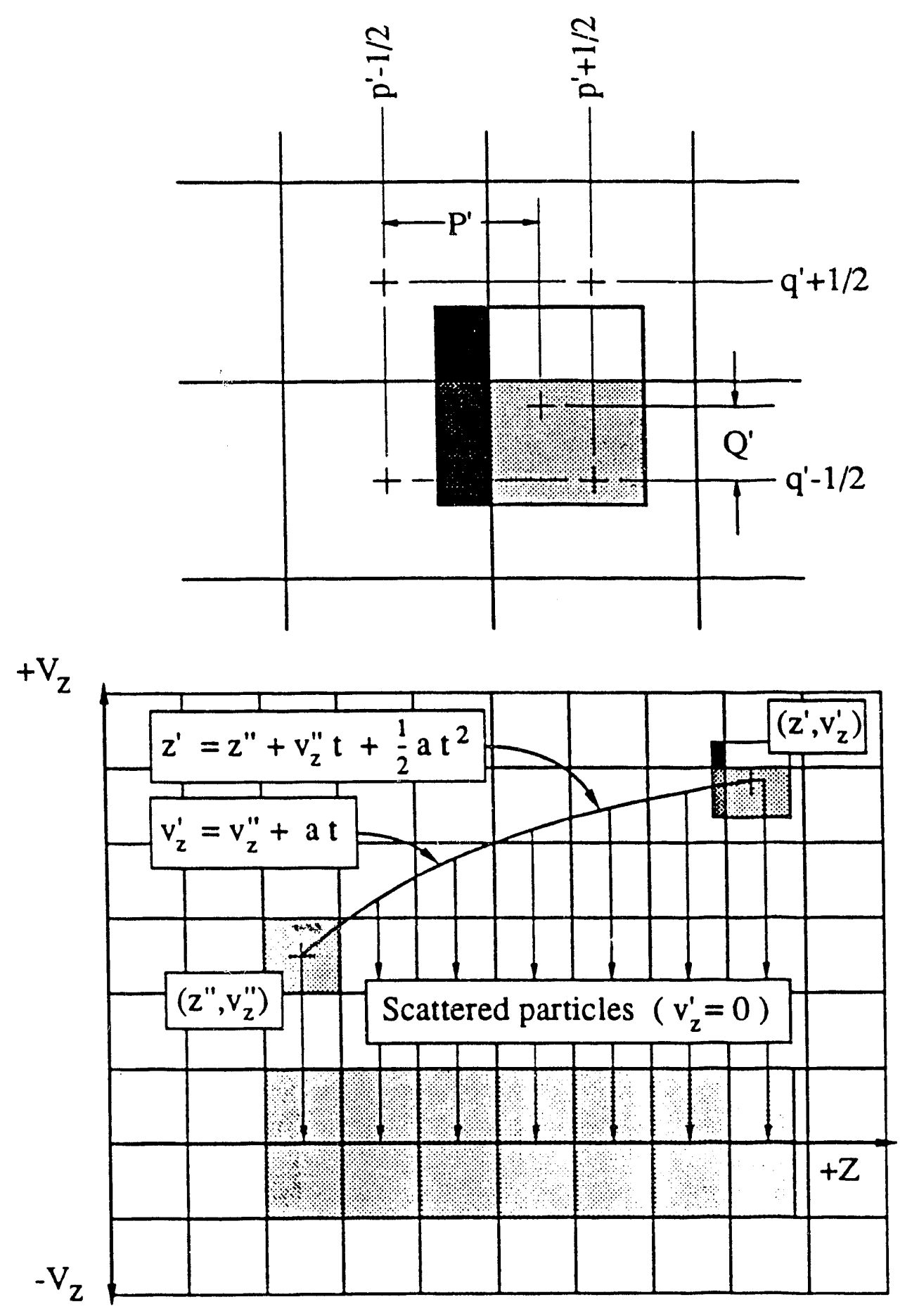

Figure II.1: Illustration of the basic method of the "Convective Scheme". 


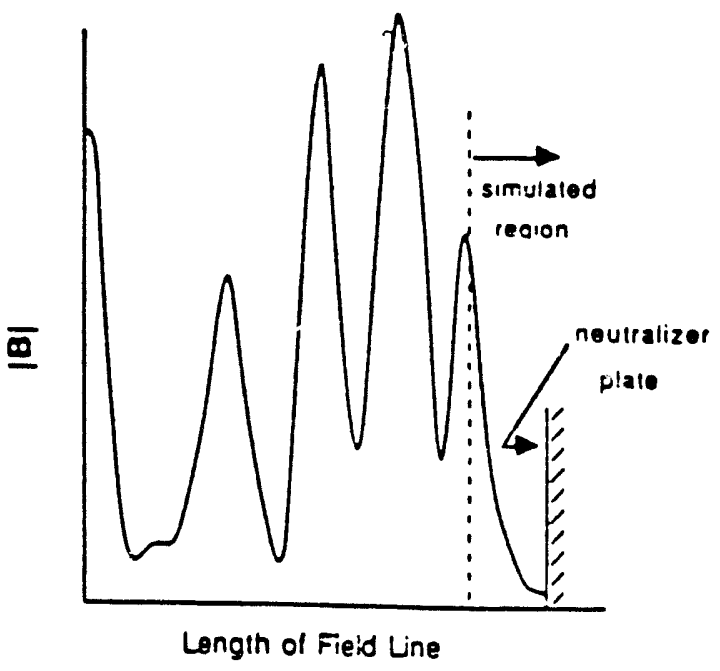

Figure III.1: Variation of the strength of $B$ along one of the ATF1 magnetic field lines. 


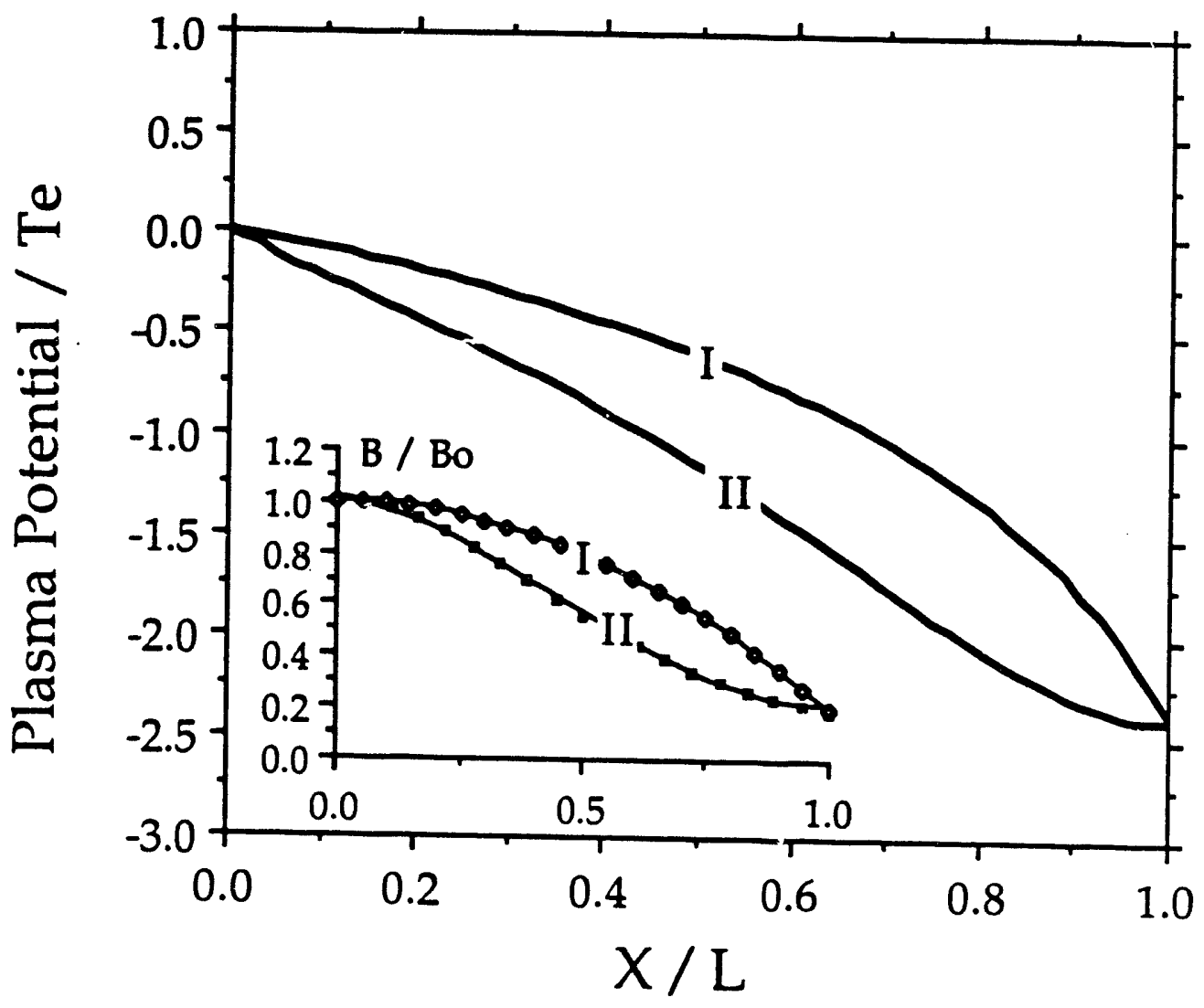

Figure III.2: The normalized potential profile resulting from a collisionless plasma flow in two different magnetic field profiles $w i t h$ the same mirror ratio at $X / L=1$. $L$ is the distance along magnetic field line in the fishtail region. 


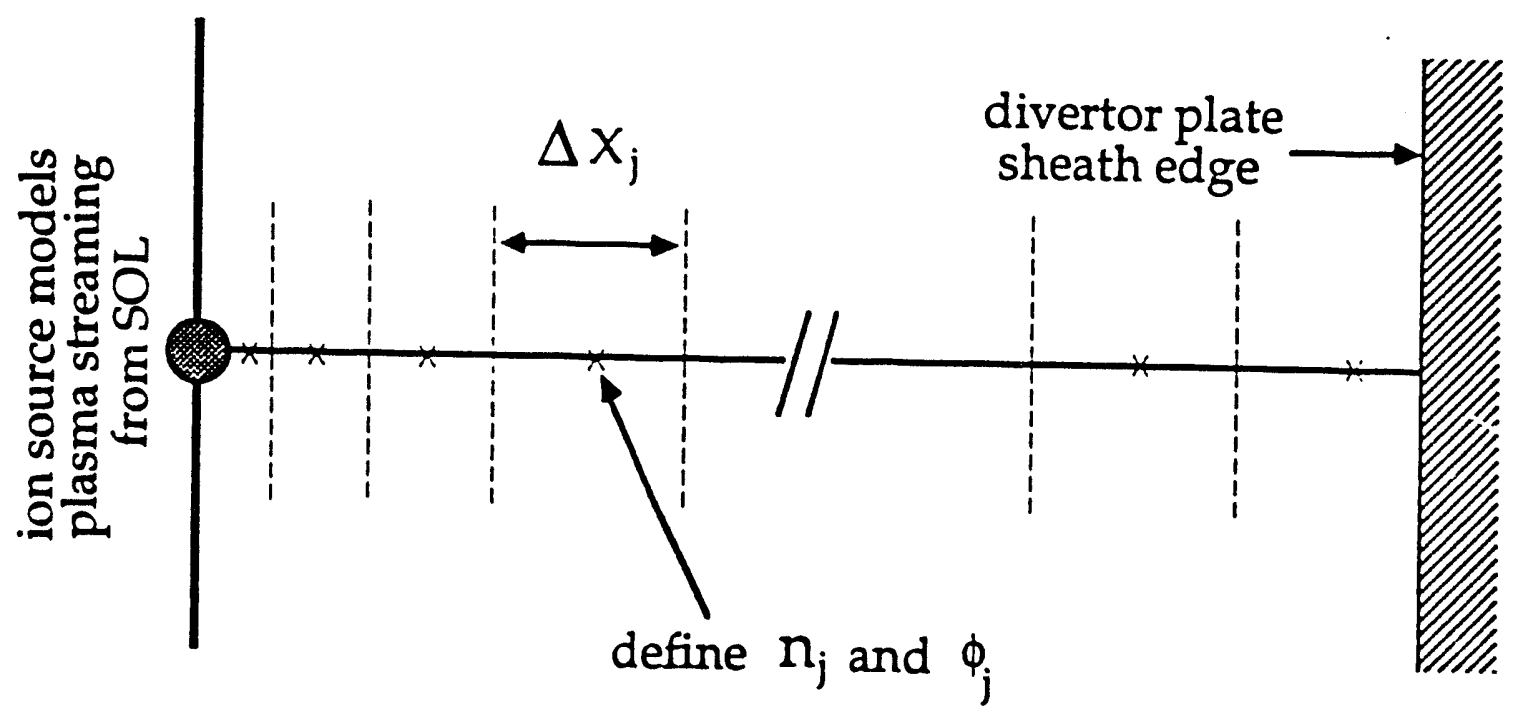

Figure III.3: The computational grid. 


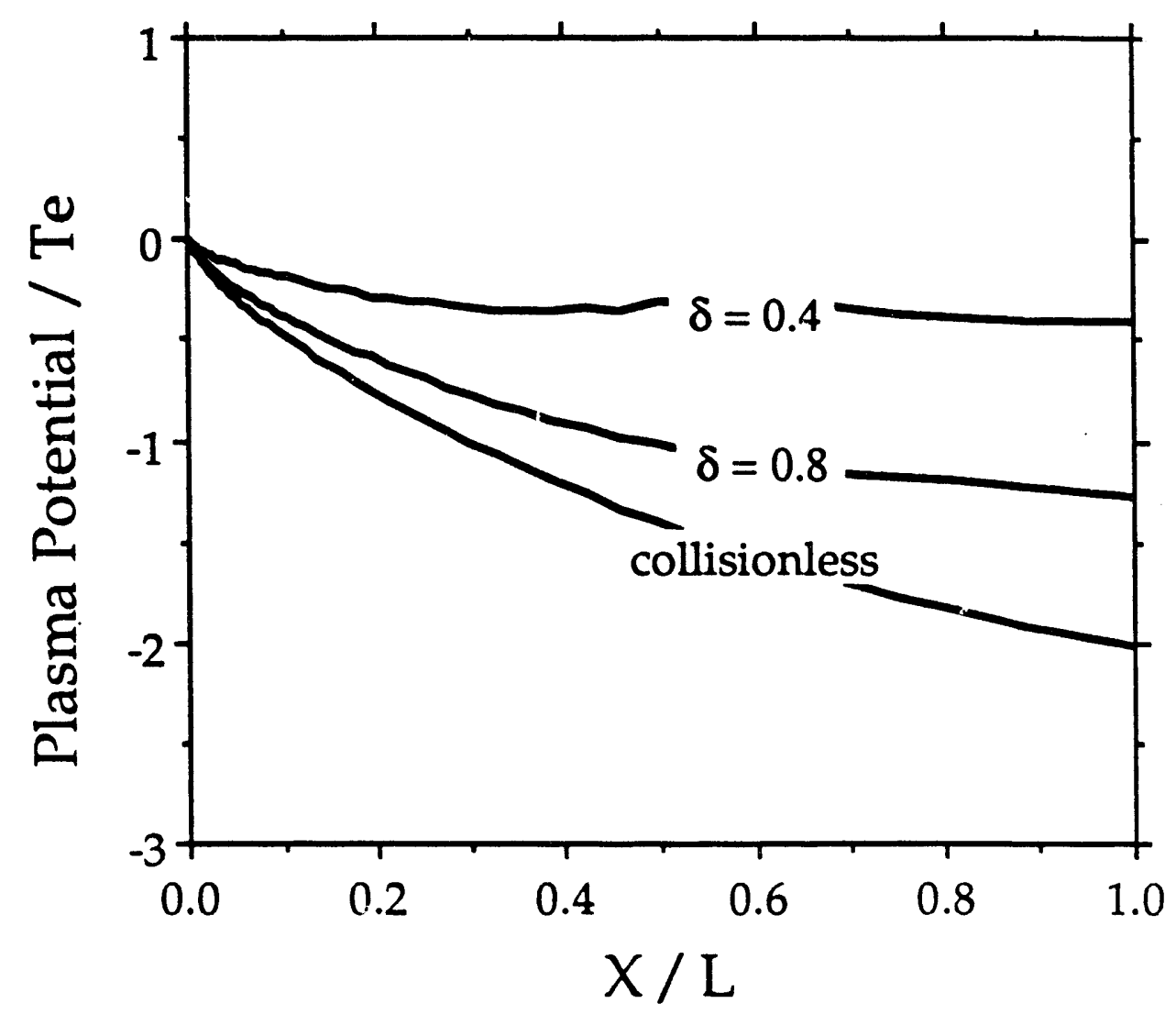

Figure III.4: Variation of the normalized plasma potential profile with collisionality. $\delta$ is the cotal ion-neutral collision mean free path normalized to the distance along the field line inside the divertor chamber, $\delta$ $=\lambda / L=0.8$. The magnetic field profile is assumed parabolic with a mirror ratio -3 . 

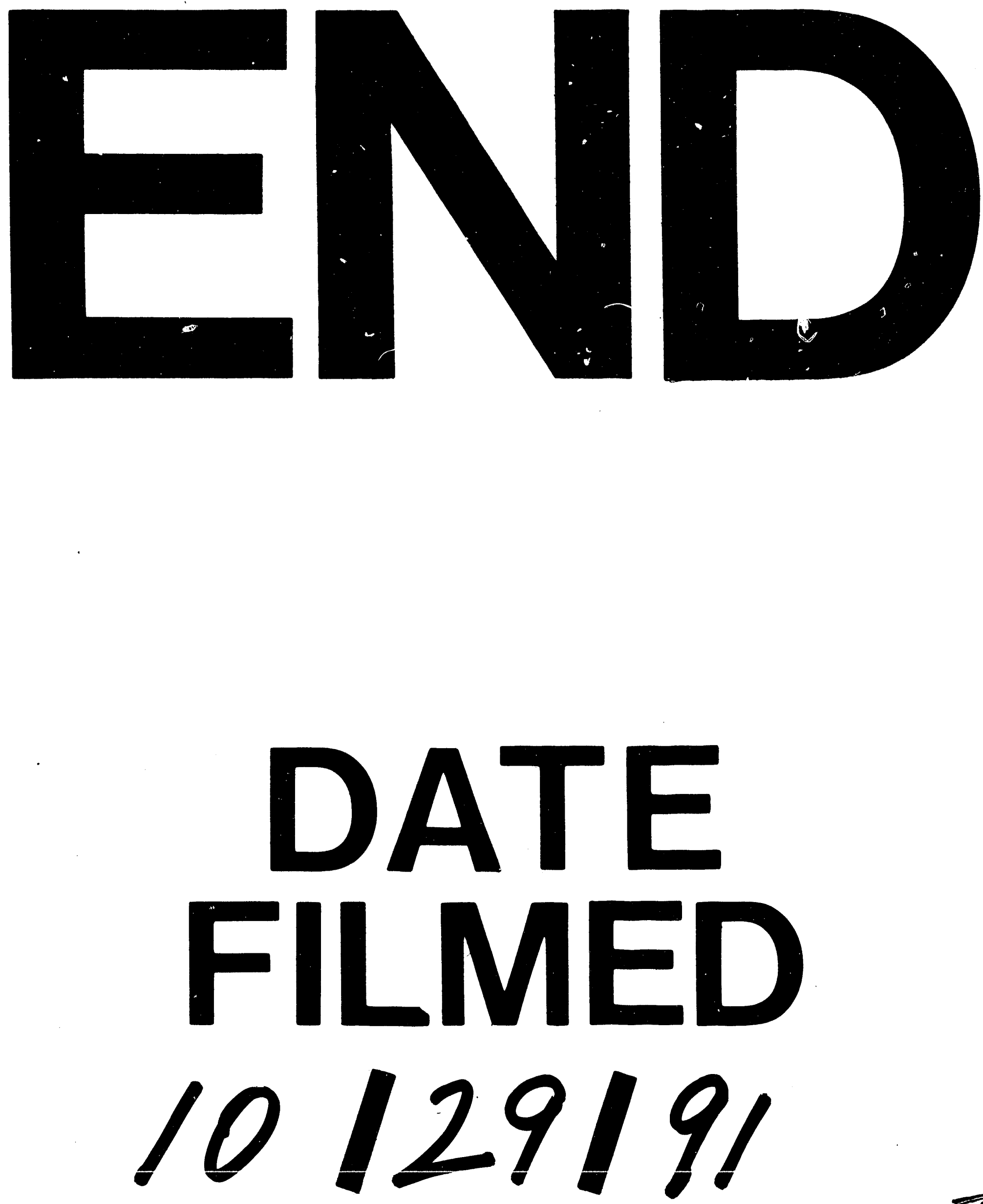
. 